\title{
Reducing the mortality from abdominal aortic aneurysms: need for a national screening programme
}

\author{
P L Harris
}

The incidence of abdominal aortic aneurysms in the British Isles as in other Western countries has risen dramatically during the second half of this century. ${ }^{1}$ Not only has there been a steady expansion in the population of elderly men, who are most at risk, ${ }^{2}$ but there is also evidence of a true increase in age specific prevalence, ${ }^{13}$ which may be related to progressive transmission of a familial predisposition to the disease. ${ }^{4}$ These trends seem set to continue.

Against this background it is a matter for serious concern that the interventions of the medical profession have not had more impact on the very high mortality associated with this condition. Lack of an effective treatment is not the problem. In the best centres elective surgical repair of abdominal aortic aneurysms carries an operative risk of under $5 \%$ and a subsequent longevity and quality of life which compares very favourably with normal expectations. ${ }^{5}$ The persistently high mortality is accounted for by the fact that a substantial majority of aneurysms remain asymptomatic until the moment of rupture, when it is usually too late for effective intervention. Of patients who reach hospital alive, between $50 \%$ and $70 \%$ can be saved by emergency surgery, but we know that many more die with a suddenness which denies them the benefit of hospital treatment. ${ }^{6}$ Often the cause of death is incorrectly diagnosed, ${ }^{6.8}$ and because precise statistics are lacking the true death rate from ruptured aneurysms is unknown. An attempt to identify all deaths from this cause within a defined community during a fixed period of time in Swansea indicated that a figure of between $80 \%$ and $90 \%$ is realistic, ${ }^{7}$ and this accords with the findings of a similar study from Stockhoim. ${ }^{8}$

\section{Screening for aneurysm}

In England and Wales, with a total population of about 47000000 , it is estimated that between 6000 and 10000 people die each year from rupture of an abdominal aortic aneurysm and that during the same period around 3000 undergo successful elective or emergency surgical treatment. ${ }^{4}$ The key to better survival is detection of the asymptomatic lesion leading to a higher proportion of elective operations, and in practice this means population screening. Pilot studies in Birmingham, ${ }^{9}$ Gloucester, ${ }^{10}$ Oxford," and Chichester ${ }^{12}$ have shown convincingly that mass

Vascular Surgical Society of Great Britain and Ireland P L Harris, secretary

Correspondence to: Mr P L Harris, Consultant Vascular Surgeon, Broadgreen Hospital, Liverpool L14 3LB.

BMF 1992;305:697-9 screening for aneurysms is not only feasible but is effective and relatively inexpensive. Furthermore, their collective experience indicates that a nationwide screening programme would be relatively easy to implement and administer.

The first requirement is the availability of a universally applicable method of diagnosis with a high degree of patient acceptability. Portable ultrasound scanners have been shown to meet these needs. B mode ultrasound imaging has long been recognised as the optimum method for diagnosing abdominal aortic aneurysms. ${ }^{13}$ An error of up to $10 \%$ associated with objective mensuration of aortic diameters and an acknowledged difficulty in determining the precise relation between the proximal limit of dilatation and the ostia of the renal arteries do not detract from the reliability with which an aneurysm can be diagnosed by a skilled operator. ${ }^{14}$ Portable machines, it seems, have retained this essential diagnostic dependability while miniaturisation has conferred other attributes not possessed by their more cumbersome predecessors. ${ }^{9-12}$ The technique of examination is non-invasive, safe, free of discomfort for the patient, and readily repeatable. The equipment can be operated by a radiographer or vascular nurse in a hospital clinic, general practitioner's surgery, or even the patient's own home, and the cost per examination is extremely low. ${ }^{10}$

\section{Population at risk and compliance}

Secondly, for mass screening to be realistic the prevalence of the disease among those screened must be high, and it is therefore important to be able to define a population at risk. Abdominal aortic aneurysm is predominantly a disease of men past middle age and is uncommon in women except for the very elderly. ${ }^{15}$ The greatest relative importance of the disease is in men aged $70-74$, in whom it accounts for $1.72 \%$ of all deaths. ${ }^{16}$ In Birmingham screening of 2669 asymptomatic men between the ages of 65 and 75 disclosed $219(8 \cdot 2 \%)$ with an aortic diameter greater than $29 \mathrm{~mm}$ and $70(2.6 \%)$ with an aortic diameter in excess of $40 \mathrm{~mm} .{ }^{9}$ Results from other studies in the United Kingdom have been broadly similar. ${ }^{10-12}$ Their collective evidence suggests that a programme to screen all apparently healthy men once, on or about their 65th birthday, will detect the majority of aneurysms. Other especially high risk groups are male siblings of patients with aneurysms, a quarter of whom also have the disease, ${ }^{1718}$ and patients who present other manifestations of chronic arterial disease-for example, those with intermittent claudication, in whom the incidence is $10-15 \% .{ }^{19}$ They require special provision for screening irrespective of any plans to screen apparently healthy people.

An important factor which can seriously undermine the value of any screening programme is low patient compliance. Experience from studies in the United Kingdom shows that response rates vary from about $50 \%$ in inner city areas to over $85 \%$ in middle class suburbs." A policy of involving local general practitioners and arranging examinations close to the patients' homes appears to contribute significantly to a high rate of compliance,,$^{20}$ and there is therefore a strong case for any future national programme to be implemented with the close involvement of local community services and family practitioner committees. Special efforts to overcome any inertia of in- 


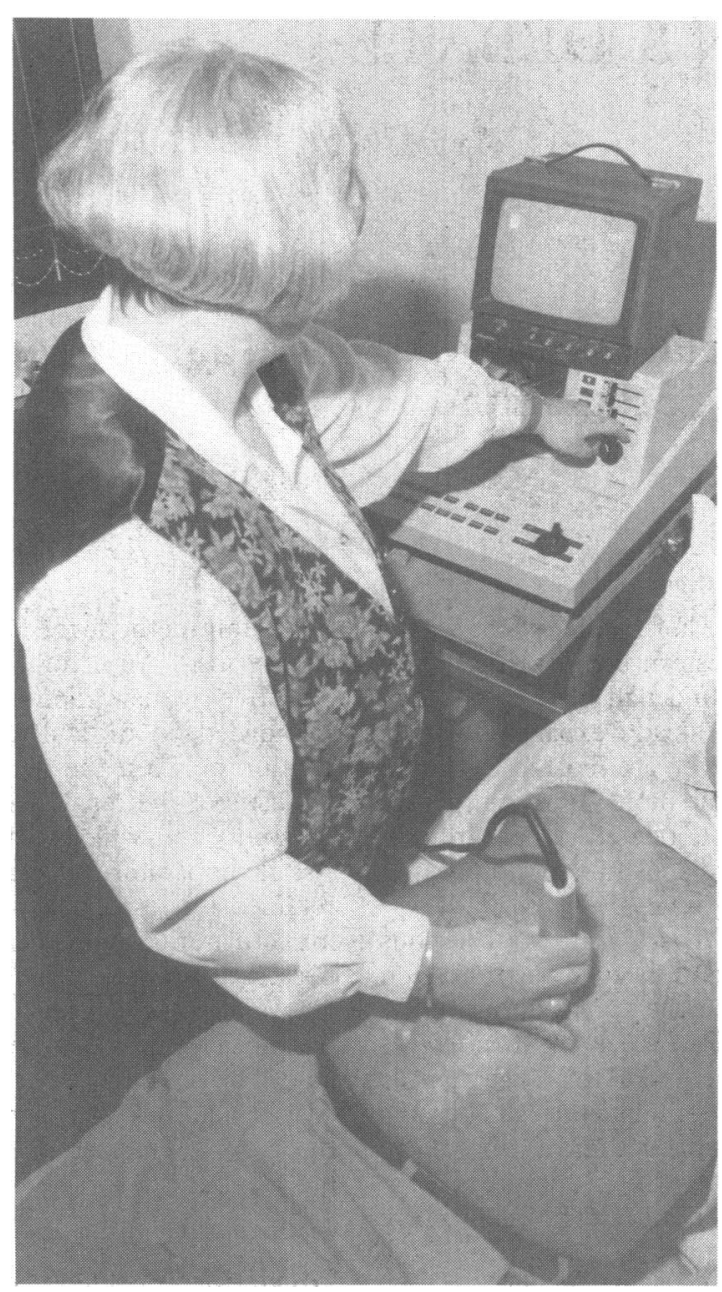

Screening for abdominal aortic aneurysm with portable ultrasound scanner

habitants of inner cities are unlikely to yield worthwhile returns. This is regrettable since it is probable that these communities include a higher proportion than average of people at risk of arterial disease and therefore abdominal aortic aneurysms. Though the effectiveness of screening would undeniably be reduced by the reticence of this important minority, the indications are that a sufficiently large majority of those at risk would be prepared to participate to ensure that a nationwide programme would yield significant benefits. ${ }^{9-12}$

\section{Treatment}

Having detected previously unsuspected disease, there must be the means available for effective treatment. A relative drawback as far as aortic aneurysms are concerned is that major surgery is required. This imposes the need for selective application. Because the risk of rupture of an aneurysm increases exponentially with its size its diameter is the main determinant of the need for treatment. Operation is justified for aneurysms of $5.5 \mathrm{~cm}$ diameter or over. In these circumstances the once only risk of definitive surgery compares very favourably with the risk of spontaneous rupture, which is cumulative in the order of $7 \%$ a year. ${ }^{21}$ Operation is not warranted initially for patients with aneurysms smaller than $4.0 \mathrm{~cm}$ diameter, in whom the risk of spontaneous rupture is very low and exceeded by the risk of death from associated ischaemic heart disease. ${ }^{22}$ These patients require regular follow up at intervals with repeated ultrasound scanning. Rather less certain is the most appropriate management policy for aneurysms with a diameter between $4 \cdot 0$ and $5 \cdot 5 \mathrm{~cm}$. A large multicentre randomised trial is under way in the United Kingdom which may resolve this problem (the United Kingdom small aneurysm trial).
For the future there is optimism that an effective endovascular alternative to conventional surgery may not be too far away. A composite stent graft device has already been deployed with success in a small number of carefully selected patients, ${ }^{23}$ and other prototypes are currently in various stages of development. All employ the same basic principles. A device which is either self expanding or can be expanded over a balloon catheter is introduced in compacted form on or within a transluminal catheter system via a remote site such as the common femoral artery. It is then positioned within the aneurysm under radiological control and released to form a lining or inner tube which will prevent further expansion or rupture of the sac. As presently envisaged, it seems likely that this type of technique will be most readily applicable to small uncomplicated aneurysms and, for reasons stated above, an extremely low complication rate is an essential prerequisite to justify active intervention for such lesions. The potential for broadening the range of patients to whom definitive treatment might be offered is, however, considerable. The successful evolution of endovascular procedures is particularly pertinent to screening programmes since two thirds of aneurysms identified by this means are under $4 \mathrm{~cm}$ diameter. ${ }^{9}$

For the present, conventional surgery represents an effective means of treating those at greatest risk of death from rupture of their aneurysm, and on the basis of the valuable information which is now available from studies conducted in the United Kingdom using this method alone, the Vascular Surgical Society is recommending that population screening for abdominal aortic aneurysms should be extended nationwide. It is believed that to do so would make an important contribution to the government's own recently declared objective of "adding years to life" as enunciated in the white paper The Health of the Nation. ${ }^{24}$ The Department of Health has been requested to make extra funding available and the initial proposal is for screening to be offered to men on or about their 65 th birthday.

\section{Economics}

The economics of population screening for abdominal aortic aneurysms on this scale have been analysed in detail by Collin..$^{25}$ The cost of screening alone would amount to less than one million pounds per annum for the whole of England and Wales or roughly $£ 100$ per aneurysm detected. An additional 4300 elective operations would be required but 1500 fewer emergency operations for ruptured aneurysms would be necessary each year. At $£ 4000$ per elective operation the net additional cost for the operations would be about $£ 8$ million. Each year around 20000 life years would be saved at a medical cost of $£ 450$ per quality adjusted life year (QALY). This compares with an estimated cost of $£ 4136$ per QALY for the national breast screening programme. ${ }^{26}$

Given the uncertain aetiology of the disease and its essentially occult nature, neither attempts at prevention nor the introduction of innovative methods of treatment can be expected to have much impact on the number of deaths from this cause. The essential requirement is to detect a higher proportion of lesions before rupture when most are asymptomatic, and from the evidence presented here it is apparent that this objective is both feasible and affordable. If it is accepted that the currently high mortality from abdominal aortic aneurysm must be reduced, then it follows that there is a need for a national screening programme.

\footnotetext{
1 Fowkes FGC, MacIntyre CCA, Ruckley CV. Increasing incidence of aortic aneurysms in England and Wales. BMF 1989;298:33-5.

2 Office of Population Censuses and Surveys. Mortality statistics, England and
} Wales. London: HMSO, 1984. 
3 Melton LJ, Bickerstaff LK, Hollier LH, Van-Peenen HJ, Lie JT, Pairolero PC, et al. Changing incidence of abdominal aortic aneurysms. A population based study. Am F Epidemiol 1984;120:379-86.

4 Powell JT, Bashir A, Dawson S. Genetic variation on chromosome 16 is associated with abdominal aortic aneurysm. Clin Sci 1990;78:13-6.

5 Collin J, Murie J, Morris PJ. The Oxford experience of surgery for abdomin aortic aneurysm. A two year prospective analysis. Surg Gynecol Obstet 1989;169:527.

1989;169:527. tip. BMF 1977;ii: 1055-7.

. (impact of vascular surgery on

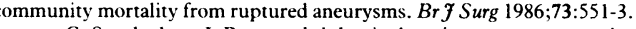
ohanson G, Swedenborg J. Ruptured abdominal aortic aneurysms: a study of incidence and mortality. Br F Surg 1986;73:101-3.

Smith FCT, Grimshaw GM, Shearman CP, Hamer JD. Aortic aneurysm screening in an urban community [abstract] Brf Surg 1992;79:358.

10 O'Kelly TJ, Heather BP. General practice-based population screening for abdominal aortic aneurysms: a pilot study. Br $\mathcal{F}$ Surg 1989;76:479.

11 Collin J, Walton J, Araujo L, Lindsell D. Oxford screening programme fo abdominal aortic aneurysm in men aged 65-74 years. Lancet 1988;ii:613-5.

2 Scott RAP, Ashton H, Kay DN. Routine ultrasound screening in managemen of abdominal aortic aneurysm. BM7 1988;296:1709.

13 McGregor JC, Pollock JG, Anton HC. The value of ultrasonography in the diagnosis of abdominal aortic aneurysm. Scott Med f 1975;20:133-7.

14 Ellis M, Powell JT, Greenhalgh RH. The limitations of ultrasonography in surveillance of small abdominal aortic aneurysms. Brf Surg 1991:78-614-6.

15 Turk KAD. The post mortem incidence of abdominal aortic aneurysm Proceedings of the Royal Society of Medicine 1965;58:869-70.
16 Office of Population Censuses and Surveys. England and Wales. Mortality statistics: cause. London: HMSO, 1986.

17 Collin J, Walton J. Is abdominal aortic aneurysm familial? BMf 1989;299:49. 18 Bengtsson $\mathrm{H}$, Norrgard $\mathrm{O}$, Angquist KA, Ekberg $\mathrm{O}$, Oberg L, Bergqvist D. Ultrasonic screening of the abdominal aorta among siblings of patients with Ultrosic

19 Bengtsson H, Ekberg D, Aspelin P, Kallero S, Bergqvist D. Ultrasound screening of the abdominal aorta in patients with intermittent claudication. Eur 7 Vasc Surg 1989:3:497-502.

20 Scott RAP, Ashton GLJ. Ultrasound screening of a general practice population for aortic aneurysm. Br $\mathcal{F}$ Surg 1986;73:318-23.

21 Szilagyi DE, Elliot JP, Smith RF. Clinical fate of the patient with asymptomatic abdominal aortic aneurysm and unfit for surgical treatment. Arch Sur 1972;104:600-6

22 Bergqvist D, Bengtsson $H$. Is screening for abdominal aortic aneurysm worthwhile? Ann Chir Gynaecol 1992;81:203-8.

23 Barodi JC, Palmaz JC, Barone HD. Transfemoral intraluminal graft implantation for abdominal aortic aneurysms. Ann Vasc Surg 1991;5:491-9.

24 Secretary of State for Health. The health of the nation. A strategy for health in England. London: HMSO, 1992.

25 Collin J. The value of screening for abdominal aortic aneurysm by ultrasound. In: Greenhalgh RM, Marrick JA, eds. The cause and management of aneurysms. London: Saunders, 1990.

26 Blamey RW, Hardcastle JD. The early detection of cancer. In: Russell RCG ed. Recent advances in surgery. 12. Edinburgh: Churchill Livingstone, 1986:29.

(Accepted 30 fuly 1992)
Institute of Medical Ethics Working Party Members of the working party are listed at the end of this report.

Correspondence to: Dr K M Boyd, Institute of Medical Ethics, Royal Infirmary of Edinburgh, Edinburgh EH3 9YW

BMF 1992;305:699-701

\title{
AIDS, ethics, and clinical trials
}

\author{
Institute of Medical Ethics Working Party on the Ethical Implications of AIDS
}

Asking a clinical scientist when he or she last read the Declaration of Helsinki' is rather like asking a seasoned airline traveller when he or she last listened to the safety announcement. The declaration sets out ethical principles which no one seriously disputes and on which other authoritative statements ${ }^{2-6}$ are essentially commentary. But the Declaration of Helsinki is sometimes more difficult to put into practice than to replicate on paper. Two of its principles, for example, mark out an area of possible moral conflict: "research involving human subjects must conform to generally accepted scientific standards," and "concern for the interests of the subject must always prevail over the interest of science and society." AIDS research highlights this possible conflict but also suggests new ways of resolving it.

\section{“RESEARCH INVOLVING HUMAN SUBJECTS MUST CONFORM TO GENERALLY ACCEPTED SCIENTIFIC STANDARDS"}

The scientific gold standard today is the randomised clinical trial. Scientists have found no more effective way to reduce bias, control variables, and establish priorities among the available options. Alternatives which have been suggested-for example, "prospective studies without randomization, but with the evaluation of patients by uninvolved third parties," or "prospective matched-pair analysis in which patients are treated in a manner consistent with their physician's views" - do not remove bias sufficiently.

\section{“CONCERN FOR THE INTERESTS OF THE SUBJECT MUST ALWAYS PREVAIL OVER THE INTEREST OF SCIENCE AND SOCIETY"}

Many patients become subjects of research from which they receive no direct benefit. The research may be non-therapeutic or, as a result of randomisation in a therapeutic clinical trial, the patient may be given a treatment which turns out to be ineffective or not given what turns out to be an effective treatment.

A subject's failure to benefit directly need not mean that concern for the interest of science and society has prevailed over concern for that of the subject. Doctors have a duty to avoid harm to their patients and to serve their best interest. But they also have a duty to respect the autonomy of patients as persons, who are the final arbiters of what is in their own best interest. A patient may have an altruistic interest in taking part in nontherapeutic research, or an interest in the possibility, however remote, of direct benefit. Informed consent means that the patient freely accepts the implications of the uncertainty principle which, scientifically as well as ethically, justifies the study.

Is it ethically justifiable to invite patients, for whose condition there is no existing treatment, to be randomised to a new treatment or a placebo? Since a clinical trial implies some chance of the new treatment being effective have these patients, in their own interest, any alternative to accepting? (British doctors, after all, have an alternative. If they judge that it is in their patient's best interest they can obtain the new treatment as an "innovative therapy." $)^{4}$

This argument seems strongest when the new treatment is designed to delay or arrest the progress of a life threatening disease. But such treatment, unfortunately, often carries the highest risks. For the patient the new treatment could result in a shorter or more distressing existence than no treatment. In some clinical trials-for example, a recent cardiac arrhythmia suppression trial $^{89}$-non-intervention groups have actually had better survival rates. Asking these patients if they are willing to be randomised to a new treatment or a placebo, then, is not offering them a choice which, in their own interests, they cannot refuse. Here again, informed consent to the implications of the uncertainty principle is the relevant ethical criterion.

\section{AIDS AND CLINICAL TRIALS IN THE UNITED STATES}

For several decades most patients have implicitly accepted the logic of the uncertainty principle and, if asked, have agreed to take part in clinical trials. But in the past few years significant numbers of patients with HIV infection or AIDS have criticised this research method, taken direct action to subvert it, and forced researchers and regulatory authorities to adopt new strategies. ${ }^{10}$ Some factors contributing to this are specific to HIV infection and AIDS, to the United States, and to those initially infected there and in Europe. What is being learnt from this experience, however, has wider application. 